Imperial/TP/93-94/56

July 1994

gr-qc/9408023

Minor revisions 10/94

\title{
The Theory of Everything vs the Theory of Anything
}

\author{
Andreas Albrecht \\ Blackett Laboratory, Imperial College \\ Prince Consort Road, London SW7 2BZ U.K.
}

\section{Introduction}

It is often stated that physicists are concerned with producing a "Theory of Everything". However, it is clear that we shall never probe all possible interactions at all possible energies. By our very nature we are limited to exploring a very special part of superspace, where spacetime behaves nice and classically, and where matter is distributed in a very orderly way. Indeed we benefit greatly from these special properties and (at least to some extent) could not survive without them. To what extent can our limited set of observations be used to pin down the specifics of a "Theory of Everything"? In the limit where the links are arbitrarily tenuous, a "Theory of Everything" might become a "Theory of Anything". A clear understanding of what we can and can not expect to learn about the universe is particularly important in the field of particle cosmology. The aim of this article is to draw attention to some key issues which arise in this context, in the hopes of fostering further discussion.

In Sect. 2 I explore the features of our own observations with suggest that a "Theory of Anything" might be the best we could hope for. I give

\footnotetext{
${ }^{1}$ To appear in the proceedings of "The Birth of the Universe and Fundamental Forces", Rome, May 1994, F. Occhionero Editor, Springer Verlag
} 
particular emphasis to the "choice of clock" issue which seems to have been under-emphasized in this context.

In Sect. 3 I discuss the important role played by posing conditional probability questions when dealing with probability distributions rather than idealized points in classical phase space. I emphasize the need to stay as far away from "anthropic" arguments as possible, but point out that quantum mechanics guarantees that we can never achieve the idealized classical view.

Section 4 explores possible implications of these ideas on inflationary cosmology. The idea that a variety of different inflaton potentials may contribute to worlds "like ours" might give a physical measure of what is "natural" for an inflation potential which is quite different than those previously used. I speculate on a possible outcome of this line of reasoning which is particularly interesting, and which removes some of the least attractive features of the inflationary cosmology.

Section 5 gives my conclusions.

\section{The inaccessability of a "Theory of Every- thing"}

Suppose there really is a "Theory of Everything" (TOE) which accurately describes nature at the most fundamental level. Would we be able to tell that this was the case based on accessible experiments? Here are some arguments which suggest that specific tests of such a theory would elude us.

\subsection{Multiple Vacua}

Many candidates for the TOE (for example superstring theory) have more than one stable (or ultra long-lived) vacuum state. Many of these states have different symmetries, or even different spacetime dimensions compared with the universe we observe. The non-commutivity of variables and their conjugate momenta ensure that no quantum state can represent a single point in classical phase space, and thus non-zero probabilities are assigned to a range of possible states Any cosmological history (starting with the high energies and densities of the big bang) would certainly assign non-trivial probabilities to the alternative vacua. Our observations would necessarily be limited to "small" excitations around a single vacuum state. Thus from our 
point of view the TOE would not predict the laws of physics we observe, but simply tell us that the laws we observe were chosen from a wide range of possibilities.

\section{$2.2 \quad$ Renormalization of Couplings}

One interesting point which has come up in the context of wormhole physics is that fluctuations in the gravitational field may renormalize the coupling constants relevant to processes we observe. In many cases the result is a continuum of non-interacting superselection sectors, each with different values for the low-energy couplings [1]. Again, the consequence is that the physical laws we observe are not specified by the TOE, but chosen from many possibilities.

\subsection{Choice of Clock}

The next two subsections are a bit outside the main flow of this article. The discussion is somewhat more technical, and I am taking the opportunity to give wider publicity to what I feel is an important ambiguity which arises in quantum cosmology. The reader who is willing to simply accept the premise that a TOE can leave a great deal of ambiguity regarding the physical laws we actually observe may wish to skip the next two subsections.

In a time reparameterization invariant theory, (such as a relativistic point particle or general relativity) the starting point for the quantum theory is a superspace with no time defined. A particular degree of freedom (or subsystem) is then assigned the role of a clock, and the evolution of the rest of the physical world as a function of time is then evaluated by inspecting the correlations between the clock subsystem and the rest of the world. (Although such a construction may seem peculiar to physicists who are used to treating time as some external parameter, the procedure just described gives a good operational definition of time as it is actually used in a laboratory.)

To sketch this construction consider a superspace $S$, with a measure defined so that given a state $|\psi\rangle_{S}$ in $S$ assigns the probability $\left.{ }_{S}\langle\psi \mid X\rangle_{S}\right|^{2}$ to

state $\left.|X\rangle_{S}\right|^{2}$. Consider a clock subsystem $C$ defined by the partition

$$
S=C \otimes R
$$


with $R$ representing the "rest" of the superspace. Part of the construction involves defining the special basis for the $C$ subspace which gives the time eigenstates of the clock. I denote this basis

$$
\left\{\left|t_{i}\right\rangle_{C}\right\}
$$

I have no qualms about labeling time (or anything else usually described by a continuous variable) using a discrete index (such as the index $i$ labeling clock eigenstates in Eq 2). The difference between a continuum and an arbitrarily closely spaced discrete label can not possibly be physically observable.

A basis $\left\{|j\rangle_{R}\right\}$ spanning the $R$ space can also be chosen, and together with the clock basis a tensor product basis spanning $S$ can be constructed. Thus a state $|\psi\rangle_{S}$ in superspace can be written

$$
|\psi\rangle_{S}=\sum_{i j} \alpha_{i j}\left|t_{i}\right\rangle_{C}|j\rangle_{R}
$$

Defining

$$
\left|\phi_{i}\right\rangle_{R} \equiv \sum_{j} \alpha_{i j}|j\rangle_{R}
$$

gives

$$
|\psi\rangle_{S}=\sum_{i}\left|t_{i}\right\rangle_{C}\left|\phi_{i}\right\rangle_{R}
$$

The state $\left|\psi\left(t_{i}\right)\right\rangle_{R}$ of subsystem $R$ at time $t_{i}$ is determined by conditioning (projecting) on clock state $\left|t_{i}\right\rangle_{C}$, giving

$$
\left|\psi\left(t_{i}\right)\right\rangle_{R}=\left|\phi_{i}\right\rangle_{R}
$$

Equation 5 tells us that "when the clock is in state $\left|t_{i}\right\rangle_{C}$ the $R$ subsystem is in state $\left|\phi_{i}\right\rangle_{R}$ (with unit probability)".

The physics of this system is contained entirely in the $\alpha_{i j}$ 's. From these one can deduce the initial state $\left|\psi\left(t_{0}\right)\right\rangle_{R}$ and the subsequent time evolution $\left|\psi\left(t_{i}\right)\right\rangle_{R}$. A different initial state evolving in a different manner would correspond to a different set of $\alpha_{i j}$ 's.

However, a different set of $\alpha_{i j}$ 's just corresponds to a different choice of clock. To see this, construct a unique integer label $k(i, j)$ for each pair $i, j$. One can then write

$$
|k\rangle_{S} \equiv|i(k)\rangle_{C}|j(k)\rangle_{R}
$$


and

$$
|\psi\rangle_{S}=\sum_{k} \alpha_{k}|k\rangle_{S}
$$

If one expands $|\psi\rangle_{S}$ in a different basis $\left|k^{\prime}\right\rangle_{S}$ one will get a different set of expansion coefficients $\tilde{\alpha}_{k^{\prime}}$. If one then identifies elements of this new basis with tensor product states labeled by $i^{\prime}\left(k^{\prime}\right)$ and $j^{\prime}\left(k^{\prime}\right)$ one gets

$$
|\psi\rangle_{S}=\sum_{i^{\prime} j^{\prime}} \tilde{\alpha}_{k^{\prime}\left(i^{\prime}, j^{\prime}\right)}\left|i^{\prime}\right\rangle_{C^{\prime}}\left|j^{\prime}\right\rangle_{R^{\prime}}
$$

Note that this amounts to the construction of a new partition $S=C^{\prime} \otimes R^{\prime}$ which in general will have nothing to do with $S=C \otimes R$.

Since bases can be chosen for $S$ which results in arbitrary $\tilde{\alpha}_{k^{\prime}}$ 's, partitions of the form $S=C^{\prime} \otimes R^{\prime}$ can be constructed which correspond to all possible

$\tilde{\alpha}_{i^{\prime} j^{\prime}}$ 's. Thus, starting with $|\psi\rangle_{S}$ one can produce all possible states evolving according to all possible time evolutions simply by choosing a suitable clock.

\subsection{Choice of Clocks: Further Discussion}

A random choice of $\alpha_{i j}$ 's will generally not give a sensible time evolution. In the general case $\left|\psi\left(t_{i}\right)\right\rangle_{R}$ and $\left|\psi\left(t_{i+1}\right)\right\rangle_{R}$ will not be related to one another by a simple Schrödinger equation. (Or, putting it another way, the Hamiltonian will have some totally random time dependence.) Thus most partitions of $S$ into $C \otimes R$ will not identify good clock subsystems.

One of the important features of our universe is that there are many subsystems which can serve equally well as clocks (which is why there can be such a thing as a clock factory, for example). That means that if one sets up a theory which accurately describes our world, there is large class of partitions $S=C \otimes R$ which simply correspond to switching from one clock to another, all of which are essentially describing the same initial conditions with the same time evolution. This fact does not conflict with the statement that there are other choices of the Clock $\otimes$ Rest partition which correspond to something totally different.

Where does the Wheeler DeWitt equation fit into my discussion? Under certain circumstances the the Hilbert space can be further partitioned into fields representing matter and gravitational degrees of freedom. If the whole system obeys Einstein gravity, one of the required constraint equations is the Wheeler DeWitt equation. Since any physical system (and many unphysical 
ones!) can be described by a suitable choice of partition $S=C \otimes R$, some will obey a suitable Wheeler DeWitt equation and many will not. Of those that do, all sorts of boundary conditions might be satisfied, and the boundary conditions would not be uniquely specified by $|\psi\rangle_{S}$.

The "problem of time" has been studied a great deal in the context of quantum gravity [2, 3]. (See for example [4, 5, 6] for work closely related to this article.) My discussion embraces the "Page-Wooters" (PW) picture of time. The ambiguity in the dynamics which I discuss here has already been noted in some specific contexts.

There are several canonical criticisms which are leveled at the sort of construction I have used here, and in particular, at PW time. Here I give my response to each:

1. Criticism: PW does not give a good definition of time because PW time is not defined in the absence of subsystems which behave like good classical clocks.

Response: I am quite happy to let time be a derived notion which has no meaning in the absence of classical clocks.

2. Criticism: Defining probability for arbitrary states in superspace (as I have) leads to normalization problems. The infinite extent of time must correspond to an undboundedness of superspace, making states in superspace non-normalizable.

Response: Since I take time to be a derived notion, which only makes sense in the presence of classical clocks, I view the idea that time is infinite with suspicion. Even a very open universe will have some probability of experiencing a quantum fluctuation which closes it, causing classical time to have finite extent.

3. Criticism: Kuchař [3] claims that one cannot construct a two-time correlation function in the PW picture.

Response: Two time correlation functions can be constructed if care is taken to make an "operational" construction which follows a realistic laboratory procedure as closely as possible. The key here is to compare records of observations made at different times rather than trying to project the poor clock on two different eigenstates (which of course gives zero). 
One possible interpretation of the result form the previous subsection is that no matter how carefully one wishes to construct one's theory of the world out of only the known physics, the theory is bound to have vast ambiguities. Even if one constructs a wavefunction describing only what we know about the world, evolving according to the observed laws of physics, there will be other partitions which describe something completely different.

The problem seems to be that the picture of a state developing in time is so far removed from a "state in superspace" that the two seem to have very little to do with one another. If a state developing in time can be interpreted as a state in superspace, than it can be interpreted as just about anything.

Another possible interpretation of the above is that I am being too loose in the way I go over to the "state in superspace". For one, I am assuming that there is an inner product defined on $S$ which allows me to perform all the manipulations required to go from one partition to another (for example, changing basis from $\left\{|k\rangle_{S}\right\}$ to $\left\{\left|k^{\prime}\right\rangle_{S}\right\}$ ). The ambiguities I mention here may be reason enough to avoid defining such a general inner product on $S$. On the other hand, just constructing a theory of the universe in the most conservative manner may already implicitly define a measure on $S$ which is suitable for all the above manipulations.

I hope the analysis presented above will form the basis for further discussions. There are issues at stake which have a great impact on how one tries to make sense of a quantum theory of the universe.

\section{Conditional Probabilities}

\subsection{General Remarks}

In the previous section I argued that processes with which we observe the world are so "superficial" relative to a TOE that uniquely specifying a TOE may impose very little constraint on the actual dynamics we observe. (Of course that raises the question of what is actually superficial, our observations or the TOE!)

The situation in which a theory presents the scientist with a set of possible alternatives (rather than a unique prediction) is not unusual in physics. A mundane example is a slowly cooled ferromagnet (in the absence of macroscopic magnetic fields). No prediction can be made of the final direction of 
the magnetization. None the less, a lot can be predicted about this system. In particular at late times one can predict that the individual spins are highly correlated, regardless of the overall ambiguity in the direction.

The correlation are exposed by asking "conditional probability" questions. Given that the spin is "up" in one corner of the magnet, one can predict that elsewhere the spins are highly likely to be pointing in the same direction. In this way one can designate certain observation to be used as "conditions" (e.g. the magnetization in the corner) and check the remaining observations (the remaining spin directions) against the predictions.

In the end, physics is about "sacrificing" the minimum number of observations for use as conditions, and using these conditions to make the maximum number of predictions.

I would like to advocate that the field of cosmology take this perspective more seriously. What are the minimum set of cosmological observations we should use as "conditions" in order to maximize our predictive power? I believe that the only guiding principle in this process is that minimum number of conditions should be used to maximum effect. Questions which can not be resolved even when all the observations are used as conditions must be classified as metaphysical questions.

Most cosmologists strongly dislike "anthropic" arguments which seem to be willing to use too many observations as conditions, and make too few predictions. Our job is certainly to get as far away from that limit as possible. It is worth remembering, however, that quantum mechanics ensures that the generic predictions will take the form of a probability distribution rather than a single point in classical phase space, so (as in the ferromagnet example) more conditions are required to produce concrete predictions.

It is particularly interesting to consider the implications of the discussion in Sect. 2 in this context. If quantum cosmology provides us with a range of possible effective dynamics, are the laws of physics at, say, the GUT scale simply a question of metaphysics? Might different domains with different effective GUT theories contribute to the physical processes we observe today? In Sect. 4 I explore the implications of this question on inflationary cosmologies. 


\subsection{The "Classical" Condition}

Before considering inflation, I wish to point to a possibly interesting "condition" at our disposal. Since the invention of quantum mechanics there has been a lot of concern focused on the extent to which classical behavior can emerge in quantum systems in the appropriate cases. Particular attention has been given to the "quantum measurement problem".

I am convinced that as long as the measurement apparatus is included in the Hilbert space, and one solves the Schrödinger equation for the whole works, there is no quantum measurement problem[7]. Even those who oppose this view would agree that the Schrödinger evolution of a realistic apparatus has very special behavior which does not reflect the properties of generic quantum systems. Given this fact, it can seem rather amazing that we are accustomed to viewing this fundamentally quantum world in such a classical manner.

It might be interesting to turn this question around, and let the classicality of the universe we observe be one of the conditions we use in cosmology (for a related line of thinking see [8]). Certainly very special initial conditions and special dynamics are required to give classical behavior. For example, one key feature required for classical behavior is a very clear statistical arrow of time [7]. This has its roots in the very low entropy nature of our initial conditions.

It is worth noting that, as emphasized by Penrose 9], the sense in which the early universe had low entropy is the sense in which the spacetime was very simple. It is these conditions which inflation is so good at producing, and indeed inflation could well be the best way of producing low entropy initial conditions. Since classical behavior seems to be so atypical of randomly chosen quantum systems, the "classical condition" may prove to be a very powerful one. It could (via the arrow of time requirement) end up "predicting" inflation, for example.

\section{Implications for Inflation}

Many different effective GUT scale theories could account for our observations of the physical world. If quantum cosmology actually forces us to consider a range of high energy physics models as equally realistic alterna- 
tives (as I argued in Sect. 2) then the implications for inflation could be very interesting 2 .

If one asks which inflaton potentials contribute the highest probability to finding a universe with whatever feature we choose to use as "conditions", what type of inflaton potentials are selected out? It is quite possible that this perspective gives quite a different notion of what is "natural" or "unnatural" about inflaton potentials. In particular, if a certain inflaton potential has an unusual functional form, but contributes much more than average to the "acceptable" regions of the universe then there is a sense in which this is a more "typical" inflaton potential.

\subsection{An Interesting Possibility}

In an inflationary model the form of the inflaton potential determines both the duration of inflation and the spectrum and amplitude of the perturbations which emerge at the end. One of the least attractive features of inflation is that there is no clear choice for the form of the inflaton potential [11, 12]. Furthermore, all of the many candidates look extremely artificial from a particle physics point of view. The less artificial versions give unacceptable predictions (such as density fluctuations so large as to destroy all the benefits of having inflation in the first place).

Given that all predictions from inflation depend on this completely unknown inflaton potential, the theory has essentially no predictive power. People try to save inflation from this fate by arguing that some inflaton potentials are more "natural" or "generic". The problem is that by the same criteria the most generic potentials give unacceptably larger perturbations.

How might the perspective presented in this article change things? The issues I have raised here may lead to a very different notion of "typical inflaton potential". In my view, the most attractive possible outcome would be if inflaton potentials giving perturbations with arbitrarily small amplitudes were to make the dominant contributions (it may be these, for example, which produce the most volume of inflated regions). In this way, any specific details of the potential would be irrelevant, and the observational predictions of inflation would be simple and unique: A perfectly flat, homogeneous, and

\footnotetext{
${ }^{2}$ Mezhlumian and Linde give an excellent modern review of the status inflation with some interesting connections to the issues raised here. 10.
} 
isotropic universe. Such inflaton potentials would certainly not be "generic" by conventional criteria, but my point is that the conventional criteria may be the wrong criteria.

The perturbations we observe can then be generated by topological defects. This picture would provide a simple explanation for why the spacetime took a specific (unperturbed) form before the defects formed. The lack of such an explanation is the greatest weakness of the standard defect scenarios. The usual attempts to combine defect scenarios with an earlier period of inflation look even more ungainly than the standard inflationary picture.

\subsection{Realistic Assessment}

How realistic is the "zero perturbation" inflationary scenario which I have just described? The simplest chaotic inflaton potentials (say $\lambda \phi^{4}$ ) do produce more expansion (more total volume) as the potential is tuned to give smaller amplitude perturbations. However, in that limit the matter density of the universe at the end of inflation is zero. Clearly any reasonable "conditions" will exclude this possibility. An attractive condition is that there is net baryon number. In models where this can only be generated at the GUT scale there will then be reheating to temperatures high enough to produce interesting defects. (In the very simplest models this may also correspond to non-negligible perturbations from inflation, however.)

Allowing the inflaton potential to take on other than the simplest forms may actually decouple the amplitude of the perturbations from the total volume of the inflated region. It may well turn out that these arguments encounter the same measure problems which appear in the standard discussions of inflation (what is the right measure to place on the space of inflaton potentials?)

It is also possible that the measure problem can be resolved, but the predictions simply do not coincide with the "zero perturbation" case I am advocating. Another interesting possibility is that the most sensible "conditions" may end up causing inflation to predict something really strange, like $\Omega \neq 1[10]$.

My conclusion, at this stage, is that these are an interesting set of ideas which require further investigation before anything concrete can be built out of them. 


\section{Conclusions}

Does it make any sense to discuss an absolutely fundamental TOE? I have suggested that even if one could construct such a theory, the observations we make are so superficial that the TOE might not constrain these observations in a very substantial way. In the limit where there is no constraint, a TOE becomes, from our perspective, a "Theory of Anything".

With so much room for ambiguity, one must be absolutely clear about the boundary between physics and metaphysics. One important tool in this effort is the posing of carefully stated conditional probability questions. We must state very carefully what we are conditioning on and what are we trying to predict. I have argued that the "classicality" condition may prove to be a very powerful one.

The zero-perturbation inflationary scenario which I have advocated here seems to suggest an elegant boundary between metaphysics and physics. In this scenario the ugly aspects of inflation (the details of the inflaton potential) are relegated to metaphysics, because the specific form of the spectrum of perturbations is unobservable. Whether this attractive scenario can be realized remains to be seen.

A clearer appreciation of the boundary between physics and metaphysics may prove very sobering for the field of cosmology. I will end, therefore, with an optimistic note: The field of cosmology has a grand history of pushing back the boundary between physics and metaphysics. Decades ago, who would have thought that the origin of relative abundances of the different chemical elements would be considered the subject of physical calculation rather than metaphysical speculation! I have no doubt that we will find more opportunities to push the boundary back even further.

I would like to thank Franco Occhionero for organizing a stimulating workshop, and I thank Neil Turok and Robert Brandenberger for useful conversations. I am also grateful for the hospitality of the Isaac Newton Institute where this manuscript was written.

After the workshop, a very interesting preprint appeared by A. Vilenkin which has considerable overlap with the ideas presented here 13. I thank A. Vilenkin for discussions of his work. 


\section{References}

[1] S. Coleman. Nucl. Phys., B308:867, 1988.

[2] C. Isham. Canonical quantum gravity and the problem of time, page 157. Kluwer, London, 1993.

[3] K. Kuchař. Time and interpretations of quantum gravity. In Proceedings of the 4th Canadian Conference on General Relativity and Relativistic Astrophysics, page 211, Singapore, 1992. World Scientific.

[4] C. Rovelli Phys. Rev D 42:2638, 1990

[5] C. Rovelli and L. Smolin Phys. Rev. Lett. 72:446, 1994

[6] M. Önder and R. Tucker Class. Quant. Grav. 11:1243, 1994

[7] A. Albrecht. Phys. Rev. D, 48:3768, 1993.

[8] M. Gell-Mann and J. B. Hartle. Quantum mechanics in the light of quantum cosmology. In W. H. Zurek, editor, Complexity, Entropy and the Physics of Information. Addison Wesley, 1990.

[9] R. Penrose. In S. W. Hawking and W. Israel, editors, General relativity: an Einstein centenary survey. Cambridge, 1979.

[10] A. Mezhlumian and A. Linde. Phys. Rev. D, page 1783, 1994. See also J Garcia-Bellido and A. Linde. Stationarity of inflation and predictions of quantum cosmology hep-th/9408023

[11] E. Kolb, M. Abney, E. Copeland, A. Liddle and J. Lidsey Reconstructing the Inflaton Potential. FERMILAB-Conf 94/189A, astro-ph/9407021

[12] E. kolb and S. Vadas Relating Spectral Indices to Tensor and Scalar Amplitudes in Inflation. FNAL-PUB-94/046-A; CfPA 94-th-14; astroph/9403001

[13] A. Vilenkin. Predictions from quantum cosmology. gr-qc/9406010, 1994. 